\title{
Study of Behavior of Digital Modulations for Beam Steerable Reflectarray Antennas
}

\author{
Fuqin Xiong \\ Cleveland State University, f.xiong@csuohio.edu \\ Robert R. Romanofsky \\ NASA Glenn Research Center, Robert.R.Romanofsky@grc.nasa.gov
}

Follow this and additional works at: https://engagedscholarship.csuohio.edu/enece_facpub

Part of the Systems and Communications Commons

How does access to this work benefit you? Let us know!

\section{Publisher's Statement}

(C) 2005 IEEE. Personal use of this material is permitted. Permission from IEEE must be obtained for all other uses, in any current or future media, including reprinting/republishing this material for advertising or promotional purposes, creating new collective works, for resale or redistribution to servers or lists, or reuse of any copyrighted component of this work in other works.

\section{Original Citation}

Fuqin, X., \& Romanofsky, R. R. (2005). Study of behavior of digital modulations for beam steerable reflectarray antennas. IEEE Transactions on Antennas and Propagation, 53, 3, 1083-1097.

\section{Repository Citation}

Xiong, Fuqin and Romanofsky, Robert R., "Study of Behavior of Digital Modulations for Beam Steerable Reflectarray Antennas" (2005). Electrical Engineering \& Computer Science Faculty Publications. 90.

https://engagedscholarship.csuohio.edu/enece_facpub/90

This Article is brought to you for free and open access by the Electrical Engineering \& Computer Science Department at EngagedScholarship@CSU. It has been accepted for inclusion in Electrical Engineering \& Computer Science Faculty Publications by an authorized administrator of EngagedScholarship@CSU. For more information, please contact library.es@csuohio.edu. 


\title{
Study of Behavior of Digital Modulations for Beam Steerable Reflectarray Antennas
}

\author{
Fuqin Xiong, Senior Member, IEEE, and Robert R. Romanofsky, Senior Member, IEEE
}

\begin{abstract}
This paper investigates the bit error rate (BER) performance of digital modulations in a system with a scanning reflectarray antenna. A reflectarray causes intersymbol interference (ISI) in a digitally modulated signal, its phase shifters' phase errors cause signal distortion, and its phase shifters' phase transient causes beam pattern degradation during direction switching. In this paper, composite signal models of the reflectarray are established for both static and transient states. Due to different feed-to-element distances and the element-to-observation distances, different delays exist in signal components. These delays cause ISI whose effect is analyzed and evaluated. Effects of phase shifters' phase errors and phase transient during beam switching are also analyzed and evaluated. Numerical calculations and simulations are performed. The analytical and simulation results for an example reflectarray at $f_{c}=26.5 \mathrm{GHz}$ and bit rate of 1.325 Gbps show that the BER degradation due to ISI is proportional to the symbol rate and the loss ranges from about $1 \mathrm{~dB}$ to around $2 \mathrm{~dB}$ in $E_{b} / N_{o}$, depending on original $E_{b} / N_{o}$, for BPSK, QPSK, 8PSK, and 16QAM. The phase error effect is negligible for lower order modulations and is unacceptable for higher order modulations such as 64QAM and 256QAM. The degradation due to phase transient effect is about $2 \mathrm{~dB}$ for BPSK and QPSK.
\end{abstract}

Index Terms-Digital modulation, reflectarray antenna.

\section{INTRODUCTION}

I N 1963, Berry introduced a new class of antennas that utilized an array of elemental radiators as a reflective source [1]. Microstrip reflectarray has been proposed as a future candidate high-gain antenna [2]. Implementing a steerable version has proven elusive, but a version based on thin film ferroelectric phase shifters has been proposed [3]. Compared to a conventional array antenna, the ferroelectric reflectarray antenna has many advantages, including: the elimination of a complex beam forming manifold (permitting an arbitrarily large aperture), relatively large device feature size thus simple lithographic fabrication, and straightforward manufacturing techniques leading to low cost. But there is an inherent intersymbol interference (ISI) problem with digital communications that comes with the way the reflectarray is operated. This is due to the fact that the antenna beam is formed by superposing reflected waves from the array elements. These waves have different delays from the elements to the receiving point. The phase shifters are designed to compensate for the modulo- $2 \pi$ phase differences so that the waves will have the same phases at the sinusoidal carrier level. However, the part of the delay that is an integer multiple of carrier period is not compensated. This causes ISI in digital modulated signals. This ISI and other effects ultimately limit the size and the gain of the reflectarray. The ISI analysis is critically needed to prove or disprove the suitability of the reflectarray antenna for high-rate digital modulated signals and to give guidance for determining antenna parameters.

The second problem associated with the reflectarray antenna is the effect of phase shifters' phase errors on the signal. The phase errors cause signal distortion, which is particularly detrimental to phase modulated signals. The third problem is the effect of phase transient of the phase shifter on the antenna beam pattern during beam switching. These two problems are also investigated in this paper.

Certain other phase shifter features are assumed; essentially that the devices behave like ideal filters. For example, we implicitly assume that the device bandwidth is sufficient. At the maximum data rate considered in this paper about a 5\% bandwidth is required. The coupled microstrip ferroelectric phase shifters described in [3] have a bandwidth of $3 \%$ to $5 \%$. But, newer designs have demonstrated bandwidths in excess of $10 \%$. Also, it is assumed that the phase shift is a linear function of frequency. These devices have indeed exhibited a linear response over bandwidths in excess of 5\%. It should also be mentioned that the ferroelectric devices are actually operated above their Curie temperature so that the films are in the paraelectric phase at room temperature. Dielectric constant variations of ferroelectric films as a function of temperature are correctable via the array control algorithm. Finally, the incident signal from the feed horn is distributed over hundreds of devices and is assumed to be sufficiently small such that the phase shifter transfer function is independent of power level.

The paper is organized as follows. Section II derives the expressions for computing the length of ISI due to feed-element distances and beam steering. Section III establishes the signal model with the above ISI, which is the static signal model when the antenna is not in switching transient. Section IV addresses the effect of static phase shifter error. Section V presents the phase shifters' characteristics and formulates the dynamic signal model with the phase transient of the phase shifters during beam switching. Section VI discusses methods of evaluating the bit error rate (BER) performance of a system with a reflectarray antenna. Simulation results of the BER performance for the static signal model (not in switching state) and the dynamic signal model (during switching) are presented in Section VII and Section VIII, respectively. Section IX concludes the paper. 


\section{DELAYS IN SignAls OF REFLECTARRAY}

RF signal from the feed illuminates hundreds of reflecting elements on the antenna panel. At the far field, the signal observed by the receiver is the sum of the reflected signals from all elements. In this composite signal, component signals have different delays, which are the sum of the delays due to different feed-element distances (called feed delay hereafter) and delays due to different radiation path lengths when the antenna beam is steered to a direction other than the bore sight (called steering delay hereafter). To make these components cophasal, modulo- $2 \pi$ compensation is provided by a phase shifter connected to each element. However, the integer multiples of the $2 \pi$-phase are not compensated for and they cause ISI.

In the following, we analyze first the feed delays and then the steering delays separately in order to assess their individual effects on the length of ISI. The results can help the antenna designers in their choice of antenna parameters. If compensation for the modulo- $2 \pi$ phase differences due to the feed delay and steering delay can be done separately, as the case may be in some designs, the total delay after modulo- $2 \pi$ compensation is just the sum of the compensated feed delay and the compensated steering delay. However, compensation for the modulo- $2 \pi$ phase differences due to feed delay and steering delay must be done jointly if only one phase shifter is used with each antenna element, as usually the case. Otherwise the total phase compensation may exceed $2 \pi$ in some cases, which is not allowed since the phase shifters are designed to compensate for less-than- $2 \pi$ phases. Therefore in Section II-C we will formulate the totaldelay expressions for the joint-compensation scheme. The results will be used in the composite signal expressions later.

The coordinate system of the reflectarray antenna for our analysis is the usual $x-y-z$ coordinates [4]. The reflecting elements (patches) are centered at the $x-y$ grid intersections. Each element is indexed by the coordinates $(m, n)$, where $m$ and $n=0, \pm 1, \pm 2, \pm 3, \ldots$ along the $x$ and $y$ axes, respectively. The center-to-center distances between two adjacent element are $d_{x}$ and $d_{y}$ along the $x$ and $y$ axes, respectively. The feed is on the $z$ axis with a distance of $F$ from the antenna plane. The direction vector $r$ points to the beam direction that is specified by the elevation angle $\theta$ and azimuth angle $\phi$.

The following analysis is based on considering the reflectarray antenna is transmitting. However, it applies to receiving mode as well since the aperture is reciprocal.

\section{A. Feed Delays}

Denote the phase of the signal received by the $m n$th element as $\varphi_{m n}$, and then the $m n$th signal is

$$
s_{m n}(t)=a_{m n} \cos \left(2 \pi f_{c} t+\varphi_{m n}\right)
$$

where $a_{m n}$ is the amplitude and $f_{c}$ is the carrier frequency. The signal received by the central element thus is

$$
s_{00}(t)=a_{00} \cos \left(2 \pi f_{c} t+\varphi_{00}\right) .
$$

For a planar antenna with the feed located on the central axis of the antenna, it is always true that the $m n$th signal is delayed in comparison with $s_{00}(t)$, i.e., $\varphi_{m n}<\varphi_{00}, m \neq 0, n \neq$ 0 . Since the phase shifters can only delay a signal rather than

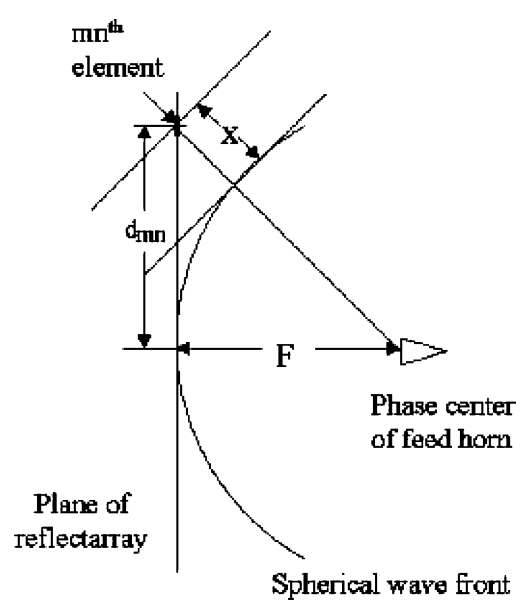

Fig. 1. Feed-to-reflectarray delays.

advance a signal, the $m n$th signal will be delayed further by $\left(2 \pi-\left(\varphi_{00}-\varphi_{m n}\right)\right)$ to make it cophasal with $s_{00}(t)$. That is, after being delayed the new phase of $s_{m n}(t)$ is

$$
\varphi_{m n}-\left(2 \pi-\left(\varphi_{00}-\varphi_{m n}\right)\right)=-2 \pi+\varphi_{00}=\varphi_{00}(\bmod 2 \pi) .
$$

This indicates that an additional carrier cycle of delay is introduced in signal $s_{m n}(t)$ by the phase shifter.

With the signal from the center of array as the reference signal, from Fig. 1, the excess delay (feed delay) of the signal $s_{m n}(t)$ relative to $s_{00}(t)$, after modulo- $2 \pi$ compensation, is given by

$\zeta_{m n c}=\left\lfloor\frac{x}{\lambda}+1\right\rfloor T_{c}=\left\lfloor\frac{\sqrt{F^{2}+d_{m n}^{2}}-F}{\lambda}+1\right\rfloor T_{c}=k_{m n} T_{c}$

where $x=$ distance difference between the distance from the feed to the $m n$th element and that from the feed to the center, $c=$ speed of light, $\lambda=$ carrier wavelength, $T_{c}=$ carrier period, $F=$ virtual focal length, $d_{m n}=$ distance from the $m n$th element to the center, $d=$ the center-to-center spacing of the array elements, $\lfloor y\rfloor=$ integer part of $y$, and $k_{m n}=$ number of carrier periods in feed delay, which is also the number of ISI cycles. Note that " +1 " accounts for the extra delay of a carrier cycle due to the nature of the phase shifter as described above.

Table I shows some values of maximum excess feed delays for $f_{c}=26.5 \mathrm{GHz}\left(\lambda=0.011313 m, T_{c}=0.03774 \mathrm{~ns}\right)$. The first row data are for a circular antenna whose layout is shown in Fig. 2 (Let us call it Antenna 1). It will be used as an example to illustrate many concepts and methods developed in this paper. The rest of the rows are for other antenna geometries. From the table, it is seen that to have a minimum feed delay, the antenna should have as small as possible an antenna size and as big as possible an F/D ratio; however too big an F/D ratio is impractical from a gain and spillover loss consideration.

\section{B. Steering Delays}

In analyzing steering delays, due to the symmetrical shape of the antenna aperture, we again use the central element of the array as the reference element and the signal reflected from this element as the reference signal $\left(s_{00}(t)\right)$. To steer the antenna beam to a direction $(\theta, \phi)$, at the bore sight, the signals 
TABLE I

MaXimum EXCESS FeEd Delays $\left(f_{c}=26.5 \mathrm{GHz}, T_{c}=0.03774(\mathrm{~ns})\right)$

\begin{tabular}{c|c|c|c|c|c}
\hline $\mathrm{F} / \mathrm{D}$ & $\mathrm{D}(\mathrm{m})$ & $x_{\max }(\mathrm{m})$ & $\zeta_{\max }(\mathrm{ns})$ & $\zeta_{\max } / T_{c}$ & $k_{\max }$ \\
\hline 1.0 & 0.2347 & 0.0277 & 0.0924 & 2.449 & 3 \\
\hline 1.0 & 0.5 & 0.0590 & 0.1969 & 5.217 & 6 \\
\hline 1.0 & 0.75 & 0.0885 & 0.2953 & 7.825 & 8 \\
\hline 1.0 & 1.0 & 0.1180 & 0.3937 & 10.43 & 11 \\
\hline
\end{tabular}

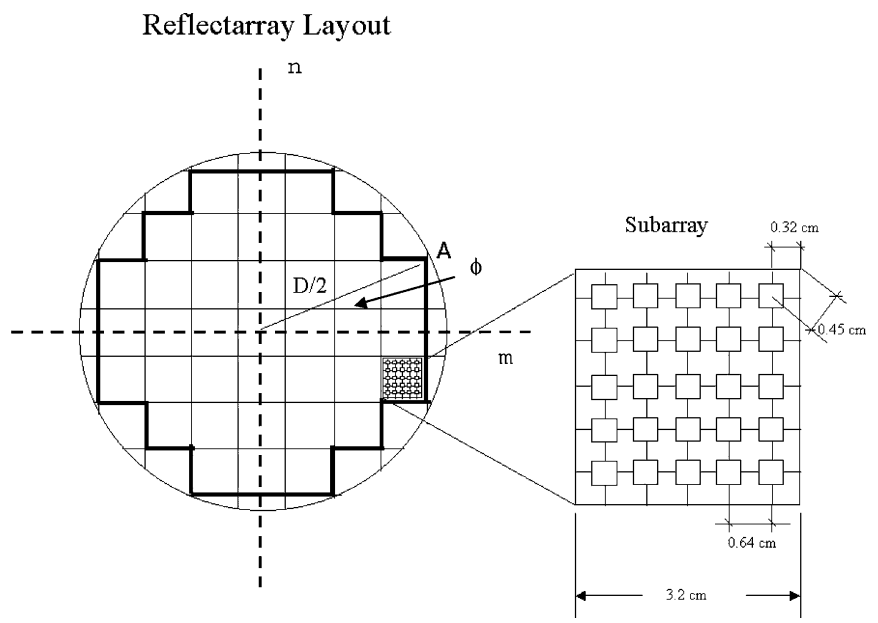

Fig. 2. Reflectarray layout for Antenna 1.

from all elements must be cophasal. Observed in the far field at the bore-sight direction, there are distance differences, hence phase differences, between signal rays reflected from antenna elements. The phase shifters are programmed to compensate for the phase differences (modulo- $2 \pi$ residual phases). We assume that the compensation method is as follows. Denote the signal's modulo- $2 \pi$ residual phase as $\varphi_{m n}$ for the signal reflected from the $m n$th element. For any beam direction other than $(0,0)$ direction, with respect to $s_{00}(t)$, there are some elements whose signals are advanced in phase $\left(\varphi_{m n}>\varphi_{00}\right)$ and some other elements whose signals are delayed in phase $\left(\varphi_{m n}<\varphi_{00}\right)$. If $\varphi_{m n}>\varphi_{00}$, the phase shifter will delay the $m n$th signal by $\left(\varphi_{m n}-\varphi_{00}\right)$ to make it cophasal with $s_{00}(t)$. If $\varphi_{m n}<\varphi_{00}$, the $m n$th signal will be delayed further by $\left(2 \pi-\left(\varphi_{00}-\varphi_{m n}\right)\right)$ to make it cophasal with $s_{00}(t)$.

The $m n$th phase shifter must compensate for the phase difference caused by $\Delta r_{m n}=r_{m n}-r_{00}$, where $r_{m n}$ and $r_{00}$ are the distances from the $m n$th element and the center (reference) element to the observation point at the far field, respectively. Depending on whether $\Delta r_{m n}$ is positive or negative, the phase compensation will be performed differently as described above. After phase compensation, the excess delay $\xi_{m n c}$ caused by $\Delta r_{m n}$ is an integer (positive or negative) multiple of the carrier period.

Now $\xi_{m n c}$ will be related to the geometrical position of each element (see Fig. 2). To make the results more general, assume that the initial phase of the signal from the $m n$th element $(m n$th signal) is $\beta_{m n}$ with $\beta_{00}=0$. Then at observation angles $(\theta, \phi)$ in the far field, the phase of the $m n$th signal would be $\psi_{m n}=$ $\alpha_{m n}+\beta_{m n}[4],{ }^{1}$ where $\beta_{m n}$ is the initial phase (controlled by the phase shifter), and

$$
\alpha_{m n}=m k_{0} d_{x} \sin \theta \cos \phi+n k_{0} d_{y} \sin \theta \sin \phi
$$

is due to distance difference $\Delta r_{m n}$, and $k_{0}=2 \pi / \lambda$ is the wave number. $\beta_{m n}$ is designed to compensate for $\varphi_{m n}=\operatorname{Mod}\left(\alpha_{m n}, 2 \pi\right)$ according to the method described before. That is

$$
\beta_{m n}= \begin{cases}-\operatorname{Mod}\left(\alpha_{m n}, 2 \pi\right), & \alpha_{m n} \geq 0 \\ -\operatorname{Mod}\left(\alpha_{m n}, 2 \pi\right)-2 \pi, & \alpha_{m n}<0 .\end{cases}
$$

Therefore

$$
\psi_{m n}= \begin{cases}\alpha_{m n}-\operatorname{Mod}\left(\alpha_{m n}, 2 \pi\right) & \\ =2 \pi l_{m n}, & \alpha_{m n} \geq 0 \\ \alpha_{m n}-\operatorname{Mod}\left(\alpha_{m n}, 2 \pi\right)-2 \pi & \\ =-2 \pi\left(l_{m n}+1\right), & \alpha_{m n}<0\end{cases}
$$

where $l_{\mathrm{mn}}$ is a positive integer.

$$
l_{m n}=\frac{\left|\alpha_{m n}-\operatorname{Mod}\left(\alpha_{m n}, 2 \pi\right)\right|}{2 \pi} .
$$

Thus the compensated delay of the $m n$th signal is

$$
\xi_{m n c}= \begin{cases}l_{m n} T_{c}, & \alpha_{m n} \geq 0 \\ -\left(l_{m n}+1\right) T_{c}, & \alpha_{m n}<0 .\end{cases}
$$

In the usual case where $d_{x}=d_{y}=d, \alpha_{m n}$ in (4) can be reduced to

$$
\alpha_{m n}=2 \pi \rho \sin \theta(m \cos \phi+n \sin \phi)
$$

where $\rho=d / \lambda$. For Antenna $1, \rho=0.566$.

Based on the geometry of the Antenna 1 shown in Fig. 2, the delays and $l_{\mathrm{mn}}$ are calculated. The maximum delays that happen at points $( \pm 17, \pm 7)$ are summarized in Table II. From the table it is seen that delays increase with the elevation angle.

\section{Joint Delays Due to Feed-Element Distances and Beam Steering}

We analyze the total delays in this section when compensation for the modulo- $2 \pi$ phase differences due to feed delay and steering delay is done jointly.

The phase of the $m n$th signal before compensation is $\alpha_{m n}$ given by (2) or (6). Thus the total phase of the $m n$th signal is in general

$$
\alpha_{m n}^{\prime}=\alpha_{m n}-\zeta_{m n} \frac{2 \pi}{T_{c}}
$$

where the - sign accounts for the fact that the feed delay $\zeta_{m n}$ is always positive (a real delay) thus it results in a negative phase

${ }^{1}$ This phase expression is given in [4] for array antennas. The reflectarray has an additional delay from the feed to the element in each signal component However, to isolate the effect of steering, we assume that feed delays are compensated for by the phase shifters in the reflectarray antenna so that the wave appears planar at the aperture. Thus the signals' phases are the same whether they are from a reflectarray or a directly radiating array fed by a manifold. 
TABLE II

Maximum Steering Delays of AnTEnNa $1\left(f_{c}=26.5 \mathrm{GHz}, T_{c}=0.03774(\mathrm{~ns})\right)$

\begin{tabular}{c|c|c|c|c|c|c}
\hline$\theta$ & $\phi$ & $m$ & $n$ & $\alpha_{m n}$ (radian) & $l_{m n}$ & $\xi_{m n c}(\mathrm{~ns})$ \\
\hline \multirow{3}{*}{$45^{\circ}$} & $0^{\circ}$ & \pm 17 & 0 & \pm 42.749 & 6 & +0.22644 \\
& & & & & & -0.26418 \\
\cline { 2 - 7 } & $22.4^{\circ}$ & \pm 17 & \pm 7 & \pm 46.232 & 7 & +0.26418 \\
& & & & & & -0.30192 \\
\cline { 2 - 7 } & $45^{\circ}$ & \pm 12 & \pm 12 & \pm 42.675 & 6 & +0.22644 \\
& & & & & & -0.26418 \\
\hline $30^{\circ}$ & $22.4^{\circ}$ & \pm 17 & \pm 7 & \pm 32.691 & 5 & +0.18887 \\
& & & & & & -0.22644 \\
\hline
\end{tabular}

of the signal. Using $\alpha_{m n}^{\prime}$ and conducting a similar derivation as in Section II-B, the compensated total delay (including feed delay and steering delay) of the $m n$th signal is found as

$$
\tau_{m n c}= \begin{cases}l_{m n}^{\prime} T_{c}, & \alpha_{m n}^{\prime} \geq 0 \\ -\left(l_{m n}^{\prime}+1\right) T_{c}, & \alpha_{m n}^{\prime}<0\end{cases}
$$

where $l_{m n}^{\prime}$ is given by

$$
l_{m n}^{\prime}=\frac{\left|\alpha_{m n}^{\prime}-\operatorname{Mod}\left(\alpha_{m n}^{\prime}, 2 \pi\right)\right|}{2 \pi} .
$$

This total delay will be used in the composite signal in the next section.

\section{Signal Model With ISI ANd EfFect of Patch Pattern AND FEED PATTERN}

A signal model of rectangular reflectarray is derived next. A circular reflectarray is approximated by arranging numerous rectangular sub-arrays together (see Fig. 2). Thus the model will be applicable to both rectangular and circular reflectarrays.

Assume the reference signal at the center of an $M \times N$ rectangular uniform array is a digitally modulated signal

$$
s_{00}(t)=A(t) \cos \left(2 \pi f_{c} t+\varphi(t)\right)
$$

where $\mathrm{A}(\mathrm{t})$ and $\varphi(t)$ jare the amplitude and phase of the signal. Any one or both of them may be information bearing. Then the total signal at observation angles $(\theta, \phi)$ in the far field is

$$
s(t)=\sum_{m=-\frac{M-1}{2}}^{\frac{M-1}{2}} \sum_{n=-\frac{N-1}{2}}^{\frac{N-1}{2}} f(m, n) p(\theta, \phi) s_{00}\left(t-\tau_{m n c}\right)
$$

where

$$
p(\theta, \phi) \approx \cos \theta
$$

is the patch's pattern, which is identical for each element, $\tau_{m n c}$ is the total delay [see (8)], and $f(m, n)$ is the signal amplitude received by the $m n$th element, which is a function of the feed's far-field pattern and the patch's pattern.

When the feed illuminates the patch array, due to the fact that the patches are on a flat surface, not on a spherical surface, we cannot use the feed's antenna pattern directly to account for the electrical field received by the patch. Rather it is a dot product of the electrical field and the unit vector along the polarization axis in the antenna plane. The result is the composite pattern function $f(m, n)$. Extending Huang's derivation in [5] for linear polarized field to circularly polarized field, we obtain the signal amplitude that can be expressed in terms of $m, n$ as [9]

$$
f(m, n)=E(r(m, n), \delta(m, n), \phi(m, n))
$$

where

$$
\begin{aligned}
& E=E(r, \delta, \phi) \approx \frac{\cos ^{q+1} \delta}{r \sqrt{2}} \\
& \times \sqrt{\left(\sin ^{4} \phi+\cos ^{4} \phi\right)\left(1+\cos ^{2} \delta\right)+4 \cos \delta \cos ^{2} \phi \sin ^{2} \phi} .
\end{aligned}
$$

For Antenna 1, $q=5.6$ in (14), and

$$
\begin{aligned}
\delta(m, n) & =\arctan \frac{d \sqrt{m^{2}+n^{2}}}{F} \\
\phi(m, n) & =\arctan \frac{n}{m} \\
r(m, n) & =\sqrt{d^{2}\left(m^{2}+n^{2}\right)+F^{2}} .
\end{aligned}
$$

Thus using (12), (8), and (13) in (11), the total signal can be written as

$$
\begin{aligned}
s(t)=(\cos \theta) & \sum_{m=-\frac{M-1}{2}}^{\frac{M-1}{2}} \sum_{n=-\frac{N-1}{2}}^{\frac{N-1}{2}} f(m, n) A\left(t-\tau_{m n c}\right) \\
& \times \cos \left[2 \pi f_{c}\left(t-\tau_{m n c}\right)+\varphi\left(t-\tau_{m n c}\right)\right] .
\end{aligned}
$$

Based on the Antenna 1 parameters, the effects of ISI of the reflectarray on the PSK signals are demonstrated using numerical calculations. Fig. 3 shows how ISI is formed due to different delays in signal components, and how ISI causes com- 


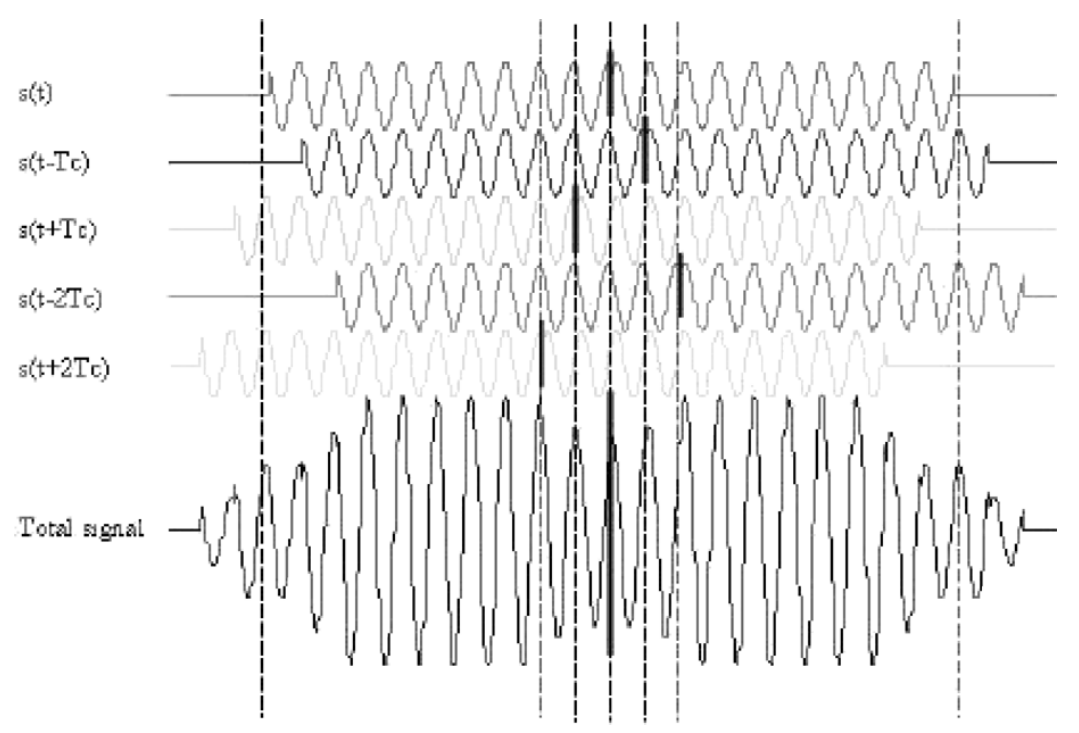

Fig. 3. Formation of ISI due to different delays in signal components.

posite signal's amplitude loss in the boundary area of two consecutive BPSK symbols ${ }^{2}$ with $180^{\circ}$ phase difference. The heavy vertical lines are symbol boundaries. In the "Total signal" waveform, the waveforms of the beginning of the first symbol and the end of the second symbol are incomplete since they are the sum of delayed waveforms of only one symbol. Therefore only the central part of the waveform around the central boundary is complete.

It is found that the larger the phases' difference between the two symbols, the larger the amplitude loss in the area around the symbol boundaries.

\section{EFFECT OF Phase ERrors of PHASE ShIFTERS}

Each phase shifter attached with each element is designed to shift the signal phase by an amount determined by the steering angles of the reflectarray antenna. However there are errors in the phase shifts. From our experimental results, the errors $(\Delta \varphi)$ are modeled as uniformly distributed in $[-\pi / 8, \pi / 8] .^{3}$ To make the results more general, we assume that the phase errors are uniformly distributed in $\left[-\Delta \varphi_{\max }, \Delta \varphi_{\max }\right]$.

With the phase error, the reflectarray composite signal can be written as

$$
\begin{aligned}
s(t)=(\cos \theta) & \sum_{m} \sum_{m} f(m, n) A\left(t-\tau_{m n c}\right) \\
& \times \cos \left[2 \pi f_{c}\left(t-\tau_{m n c}\right)+\varphi\left(t-\tau_{m n c}\right)+\Delta \varphi\right] .
\end{aligned}
$$

The average of this signal is easily found to be

$$
\begin{aligned}
E\{s(t)\} & =(\cos \theta) \sum_{m} \sum_{m} f(m, n) A\left(t-\tau_{m n c}\right) \\
& \left.\times \frac{\sin \Delta \varphi_{\max }}{\Delta \varphi_{\max }} \cos \left[2 \pi f_{c}\left(t-\tau_{m n c}\right)+\varphi\left(t-\tau_{m n c}\right)\right]\right\}
\end{aligned}
$$

${ }^{2} \mathrm{~A}$ symbol is a burst of the sinusoidal carrier signal whose amplitude and phase are determined by a baseband digital pulse.

${ }^{3}$ This distribution is ensured since all the phase shifters used in the development of the reflectarray were screened on-wafer and only those devices that fall within $\pm 22.5^{\circ}$ of some basis phase shifter are incorporated into the array. This is not an uncommon practice. where $E(x)$ is the expectation of $x$. This shows that the averaged effect of phase error is to introduce an amplitude loss of

$$
L_{\Delta \varphi}=\frac{\sin \Delta \varphi_{\max }}{\Delta \varphi_{\max }} \text { or } L_{\Delta \varphi}(\mathrm{dB})=|20 \log | \frac{\sin \Delta \varphi_{\max }}{\Delta \varphi_{\max }}||
$$

and there is no net phase shift of the composite signal due to the phase errors. For the Antenna 1 with a maximum phase error of $\pi / 8$, the loss is only $0.224 \mathrm{~dB}$. From (19) it can be seen that the phase error loss is a sinc function of $\Delta \varphi_{\max }$ and it can be shown that the maximum phase error must be less than 47.06 to limit the loss within $1 \mathrm{~dB}$.

However, despite that there are hundreds of component signals in the composite signal, the real composite signal will have not only amplitude loss, but also small net phase error. The small net phase error will have a degrading impact on the BER performance of the high-order PSK schemes and QAM schemes, since the phase differences between constellation points are very small. This will be demonstrated by the simulation results.

\section{EFFect of Phase Transient}

All above discussions are about ISI and phase errors in the static state of the antenna. The situation is different when the antenna beam is switching from one direction to another. In this section, the effect of phase transient of the phase shifters during beam switching is studied.

The reflectarray beam direction can be switched in two modes: parallel or serial. In parallel mode, the phase shifters' phase settings are updated simultaneously. Since different control voltages must be applied to each phase shifter, the times needed to make those changes are therefore different. In other words, phase changes may be started simultaneously but cannot finish simultaneously. In serial mode, the phase shifters' phase settings are updated one by one. In both cases, the antenna pattern will evolve from the original position to a new position. During the process, the antenna pattern actually undergoes a shape evolution. Distortion and loss will occur during the 
switching. The disadvantage of serial switching is that the total switching time is much longer than that of parallel switching, since it is the sum of switching times of all phase shifters. Thus parallel switching is the preferable mode and it is assumed in the rest of this discussion.

\section{A. Phase Adjustments for Beam Switching}

Consider joint modulo- $2 \pi$ phase compensation for feed delay and steering delay. To switch from direction $\left(\theta_{0}, \phi_{0}\right)$ to a new direction $(\theta, \phi)$, from (3), the phase shift of the $m n$th element is

$$
\beta_{m n}(\theta, \phi)= \begin{cases}-\operatorname{Mod}\left[\alpha_{m n}^{\prime}(\theta, \phi), 2 \pi\right], & \alpha_{m n}^{\prime}(\theta, \phi) \geq 0 \\ -\operatorname{Mod}\left[\alpha_{m n}^{\prime}(\theta, \phi), 2 \pi\right]-2 \pi, & \alpha_{m n}^{\prime}(\theta, \varphi)<0\end{cases}
$$

where $\alpha_{m n}^{\prime}(\theta, \phi)$ is given by (7).

The phase change is shown in (21), at the bottom of the page. Assuming $d_{x}=d_{y}=d$, define $\rho=d / \lambda$, then

$$
\begin{aligned}
\Delta \alpha_{m n}^{\prime}=\alpha_{m n}^{\prime}(\theta, \phi)-\alpha_{m n}^{\prime}\left(\theta_{0}, \phi_{0}\right) \\
=2 \pi \rho\left[m\left(\sin \theta \cos \phi-\sin \theta_{0} \cos \phi_{0}\right)\right. \\
\left.\quad+n\left(\sin \theta \sin \phi-\sin \theta_{0} \sin \phi_{0}\right)\right] .
\end{aligned}
$$

It can be seen from (21) and (22) that the phase change of each element is a function of the element location $(m, n)$. Thus, in general, each phase shifter's phase setting is changed by a different amount to maintain a constant phase gradient in $x$ and $y$. Also, the phase change depends on the changes in $(\theta, \phi)$. By exhaust computer search, it is found that for a $35 \times 35$ square array (which encompasses the 925 elements of the circular Antenna 1 ) with $\rho=0.566$, among $35 \times 35=1225$ elements, about $1 \%$ has a phase adjustment that is more than $270^{\circ}$. The search has considered all possible $(\theta, \phi)$ directions from $\left(0^{\circ}, 0^{\circ}\right)$ to the maximum $\left(44^{\circ}, 360^{\circ}\right)$ in steps of a multiple of $2^{\circ}$ in azimuth or elevation or both.

\section{B. Phase Shifter Characteristic}

The steady-state characteristic of the phase shifter for Antenna 1 is shown in Fig. 4. The control voltage ranges from $0 \mathrm{~V}$ to $\approx 400 \mathrm{~V}$ to induce reflection mode phase changes from $0^{\circ}$ to $360^{\circ}$ (i.e., $0^{\circ} \leq \angle S_{11} \leq 360^{\circ}$ ). A model for the phase shifter operation was provided in [10]. The steady-state characteristic is very accurately curve-fit by the following polynomial relating phase shift $\psi$ to control voltage $v$

$$
\psi(v)=c_{0}+c_{1} v+c_{2} v^{2}+c_{3} v^{3}+c_{4} v^{4}
$$

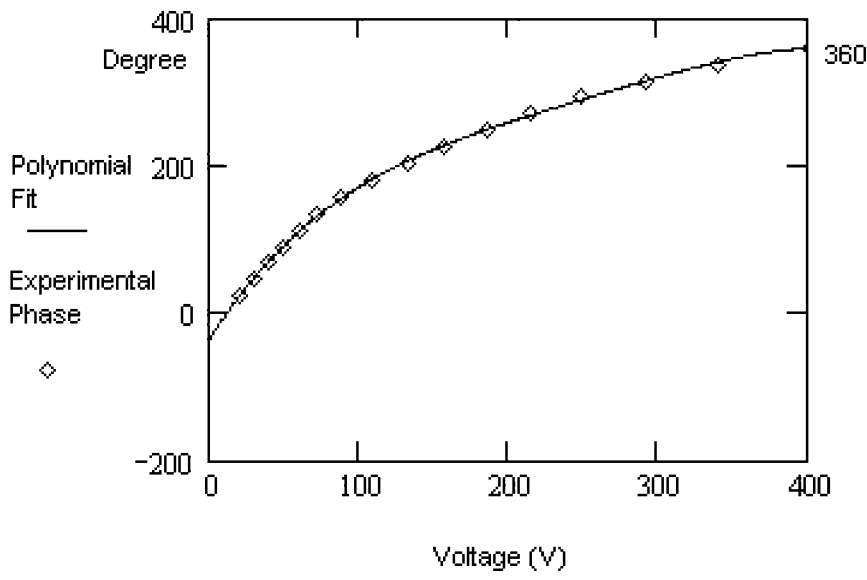

Fig. 4. Steady-state characteristic of phase shifter.

where the coefficients are given by

$$
\begin{aligned}
\mathbf{c}^{T}= & {\left[\begin{array}{lllll}
c_{0} & c_{1} & c_{2} & c_{3} & c_{4}
\end{array}\right] } \\
= & {\left[\begin{array}{lll}
-35.114 & 3.179 & -0.014 \\
& & 3.696 \times 10^{-5} \\
& -3.607 \times 10^{-8}
\end{array}\right] . }
\end{aligned}
$$

The relative mean square fitting error is $1.881 \times 10^{-4}$ for the entire range of the voltage and $3.162 \times 10^{-4}$ for $v \leq 108 \mathrm{~V}$.

The transient characteristic of the phase shifter's control voltage is shown in Fig. 5, and it dominates the overall response. That is, the measured rise time of a typical ferroelectric phase shifter is less than 1 ns and negligible by comparison. This is not surprising since the ferroelectric polarization mechanism requires the dipoles to reverse direction at the rate of the microwave signal. And, phase shifters have already been demonstrated above $K$-band. The curve in Fig. 5 can be approximated by

$$
v(t)=V_{M}\left(1-e^{-\frac{t}{\tau}}\right)=444\left(1-e^{-\frac{t}{0.0025}}\right)
$$

where $V_{M}$ is the maximum voltage and $\tau$ is a time constant. The relative mean square fitting error is $2.585 \times 10^{-3}$ for the entire range of the voltage and $7.349 \times 10^{-4}$ for the first $5 \mathrm{~ms}$. By substituting (25) for $v$ in (23), the composite characteristic of the phase transient of the phase shifter is obtained as

$$
\psi(v(t))=c_{0}+c_{1} v(t)+c_{2} v^{2}(t)+c_{3} v^{3}(t)+c_{4} v^{4}(t) \triangleq \psi(t)
$$

$$
\begin{aligned}
\Delta \beta_{m n}(\theta, \phi) & =\beta_{m n}(\theta, \phi)-\beta_{m n}\left(\theta_{0}, \phi_{0}\right) \\
= & \left\{\begin{array}{lr}
-\operatorname{Mod}\left\{\Delta \alpha_{m n}^{\prime}, 2 \pi\right\}, & \alpha_{m n}^{\prime}(\theta, \phi) \geq 0 \& \alpha_{m n}^{\prime}\left(\theta_{0}, \phi_{0}\right) \geq 0 \\
-\operatorname{Mod}\left\{\Delta \alpha_{m n}^{\prime}, 2 \pi\right\}+2 \pi, & \alpha_{m n}^{\prime}(\theta, \phi) \geq 0 \& \alpha_{m n}^{\prime}\left(\theta_{0}, \phi_{0}\right)<0 \\
-\operatorname{Mod}\left\{\Delta \alpha_{m n}^{\prime}, 2 \pi\right\}-2 \pi, & \alpha_{m n}^{\prime}(\theta, \phi)<0 \& \alpha_{m n}^{\prime}\left(\theta_{0}, \phi_{0}\right) \geq 0
\end{array}\right.
\end{aligned}
$$




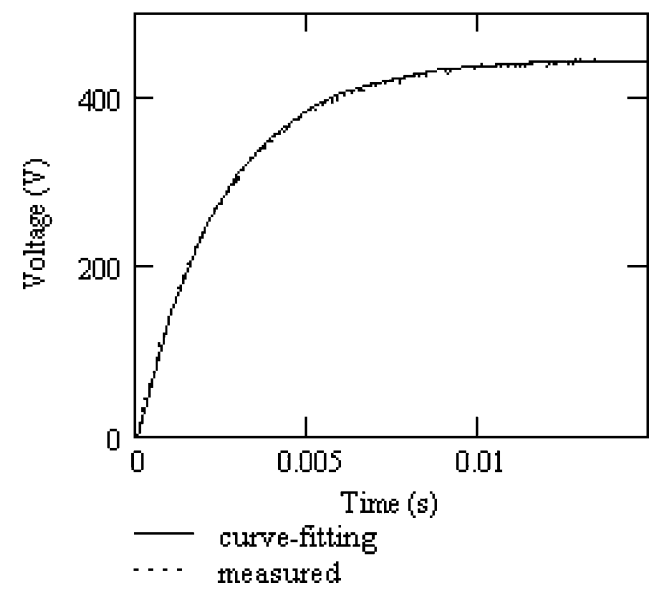

Fig. 5. Transient response of the phase shifter's control voltage.

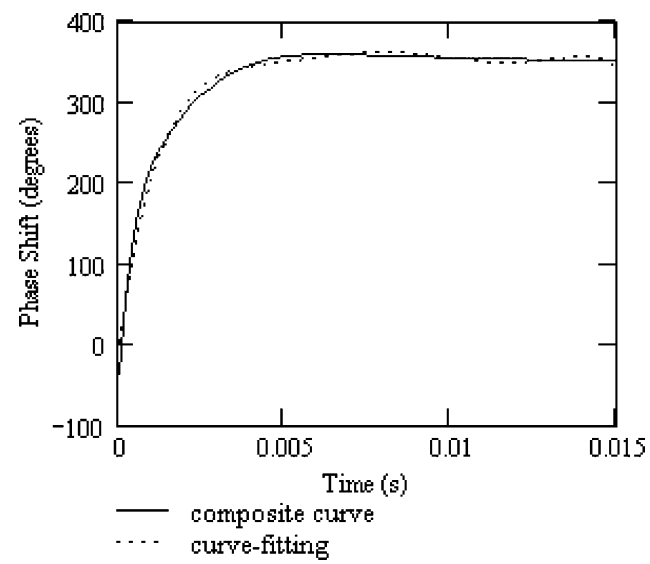

Fig. 6. Transient response of the phase shifter's phase.

which is shown in Fig. 6. This curve can be fit with a sixth order polynomial with reasonable accuracy

$$
\psi(t)=s_{0}+s_{1} t+s_{1} t^{2}+s_{3} t^{3}+s_{4} t^{4}+s_{5} t^{5}+s_{6} t^{6}
$$

where the coefficients are given by

$$
\begin{aligned}
& \mathbf{s}^{T}=\left[\begin{array}{lllllll}
s_{0} & s_{1} & s_{2} & s_{3} & s_{4} & s_{5} & s_{6}
\end{array}\right]
\end{aligned}
$$

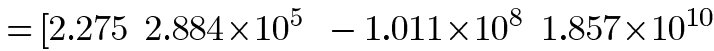

$$
\begin{aligned}
& \left.-1.835 \times 10^{12} 9.17 \times 10^{13}-1.813 \times 10^{15}\right] \text {. }
\end{aligned}
$$

The relative mean square fitting error is 0.016 for the entire range of the voltage and 0.044 for the first $5 \mathrm{~ms}$.

\section{Effect of Phase Shifters on Beam Evolution}

One way to assess the effect of phase transient is to examine the array factor's evolution during beam switching. The static phase random errors can also be conveniently included in the array factor. However, ISI cannot be included in the array factor since the array factor is derived for unmodulated sinusoidal signals. Therefore, for digitally modulated signals, the degradation obtained from array factor evolution during beam switching is the extra degradation on top of the effect of ISI. To assess the total effect of all three impairments, Monte Carlo simulation is used. The signal model for simulation is developed in the next section.
In this section, we only consider the impact on power loss as the beam is scanning. Phase shifters' errors are also included. The array factor is given by the following:

$$
\begin{aligned}
& A F(\theta, \phi) \\
& =\sum_{n} \sum_{m} e^{j(m-1) \psi_{x}\left(\theta, \phi, \theta_{0}, \phi_{0}\right)} e^{j \Delta \varphi} e^{j(n-1) \psi_{y}\left(\theta, \phi, \theta_{0}, \phi_{0}\right)} e^{j \Delta \varphi}
\end{aligned}
$$

where $\Delta \varphi \in[-\pi / 8, \pi / 8]$ represents the random phase errors of the phase shifters, and

$$
\begin{aligned}
& \psi_{x}\left(\theta, \phi, \theta_{0}, \phi_{0}\right)=k d_{x} \sin \theta \cos \phi+\beta_{x}\left(\theta, \phi, \theta_{0}, \phi_{0}\right) \\
& \psi_{y}\left(\theta, \phi, \theta_{0}, \phi_{0}\right)=k d_{y} \sin \theta \sin \phi+\beta_{y}\left(\theta, \phi, \theta_{0}, \phi_{0}\right)
\end{aligned}
$$

are the total phases after compensated by

$$
\begin{aligned}
\beta_{x}(\theta, & \left.\phi, \theta_{0}, \phi_{0}\right) \\
= & -k d_{x} \sin \theta \cos \phi \\
& +\left[\left(-k d_{x} \sin \theta_{0} \cos \phi_{0}\right)-\left(-k d_{x} \sin \theta \cos \phi\right)\right] \\
& \times\left[1-\exp \left(\frac{-t}{\tau}\right)\right] \\
\beta_{y}\left(\theta, \phi, \theta_{0}, \phi_{0}\right) & \\
= & -k d_{y} \sin \theta \sin \phi \\
& +\left[\left(-k d_{y} \sin \theta_{0} \sin \phi_{0}\right)-\left(-k d_{y} \sin \theta \sin \phi\right)\right] \\
& \times\left[1-\exp \left(\frac{-t}{\tau}\right)\right] .
\end{aligned}
$$

These terms represent the transient phase compensations during switching from direction $\left(\theta_{0}, \phi_{0}\right)$ to direction $(\theta, \phi)$. The first term is the compensation when the beam is eventually at the new direction and the second term is the changing phase when the phase shifter is updating its phase. The factor $\left[1-e^{-t / \tau}\right]$ approximates the transient characteristic of the phase shifter, where $\tau$ is a random number which represents the random nature of the phase shifter status. In this simulation, to simplify the simulation and to save time, we have not used the phase transient given in (27). In stead the above approximation is used.

For the array under consideration here the beam width is on the order of $5^{\circ}$. So a typical update will not require the beam to scan more than $5^{\circ}$ otherwise the target would fall outside the field of view. From Fig. 6 and statistics of phase changes let us assume for convenience that an average phase shifter responds in $0.3 \mathrm{~ms}$ and that $6.25 \%$ ( 1 out of 16 ) require $0.7 \mathrm{~ms}$. In addition we account for phase errors distributed uniformly over $[-\pi / 8, \pi / 8]$. Under these circumstances it can be shown by numerical calculation that for a $5^{\circ} \theta$ and $\phi$ scan the gain falls off by $\approx 36 \%$ ( $1.93 \mathrm{~dB}$ loss). These results are significant but not catastrophic. Apparently, the integrity of the main beam is largely preserved due to an averaging effect of the transient response instead of completely dissipating due to arbitrary phase settings.

\section{Signal Model During Switching}

We assume that the phase shifters' phase settings are updated simultaneously. Since each phase shifter needs a different phase adjustment, different control voltages must be applied to different phase shifters. The times needed to make those changes are therefore different. In other words, phase changes may be 
started simultaneously but cannot finish simultaneously. In addition, since the initial steady-state phases are different for different phase shifters, the phase adjustments will follow different segments of the phase transient response curve in Fig. 6.

Before the beam switches, the phase shifter of each element has a phase setting of $\beta_{m n}\left(\theta_{0}, \phi_{0}\right)$ which is set by the control voltage $v_{m n}\left(\theta_{0}, \phi_{0}\right)$. After switching to the new direction $(\theta, \phi)$, the phase shifter has a phase setting of $\beta_{m n}(\theta, \phi)$ which is set by the control voltage $v_{m n}(\theta, \phi)$. When the phase setting is $\beta_{m n}\left(\theta_{0}, \phi_{0}\right)$ or $\beta_{m n}(\theta, \phi)$, the signal phase will only be the phase due to the data, no extra phase due to the travel distances of the signal waves shows up, since it has been compensated by $\beta_{m n}\left(\theta_{0}, \phi_{0}\right)$ or $\beta_{m n}(\theta, \phi)$. However, during switching transient, $v_{m n}\left(\theta_{0}, \phi_{0}\right)$ evolves to $v_{m n}(\theta, \phi)$. Correspondingly, $\beta_{m n}\left(\theta_{0}, \phi_{0}\right)$ evolves to $\beta_{m n}(\theta, \phi)$. This transient phase setting $\beta_{m n}\left(\theta_{0}, \phi_{0}, \theta, \phi, t\right)$ is a value between $\beta_{m n}\left(\theta_{0}, \phi_{0}\right)$ and $\beta_{m n}(\theta, \phi)$, and it is determined by the transient voltage $v_{m n}\left(\theta_{0}, \phi_{0}, \theta, \phi, t\right)$ that is given by

$$
\begin{aligned}
\left.v_{m n}\left(\theta_{0}, \phi_{0}, \theta, \phi, t\right)=V_{M}\left[1-\exp \left\{-\frac{t+t_{0 m n}}{\tau}\right\}\right]\right] \\
=444\left[1-\exp \left\{-\frac{t+t_{0 m n}}{0.0025}\right\}\right], \\
0 \leq t \leq t_{m n}-t_{0 m n}
\end{aligned}
$$

where the starting time of switching is set to 0 and $\left(t_{m n}-t_{0 m n}\right)$ is the time needed for the $m n$th element to complete the switching; $t_{0 m n}$ corresponds to starting control voltage $v_{m n}\left(\theta_{0}, \phi_{0}\right)$, and $t_{m n}$ corresponds to ending control voltage $v_{m n}(\theta, \phi)$. In other words, $v_{m n}\left(\theta_{0}, \phi_{0}, \theta, \phi, t\right)$ will follow a segment of the curve in Fig. 5 starting from a point $t_{0 m n}$ on the time axis, ending at a point $t_{m n}$ on the time axis. Since

$$
v_{m n}\left(\theta_{0}, \phi_{0}\right)=V_{M}\left[1-\exp \left\{-\frac{t_{0 m n}}{\tau}\right\}\right]
$$

it can be solved to give

$$
\begin{aligned}
t_{0 m n} & =-\tau \ln \left[1-\frac{v_{m n}\left(\theta_{0}, \phi_{0}\right)}{V_{M}}\right] \\
& =-0.0025 \ln \left[1-\frac{v_{m n}\left(\theta_{0}, \phi_{0}\right)}{444}\right] .
\end{aligned}
$$

Since the ending control voltage is $v_{m n}(\theta, \phi)$, the ending time of switching is

$t_{m n}=-\tau \ln \left[1-\frac{v_{m n}(\theta, \phi)}{V_{M}}\right]=-0.0025 \ln \left[1-\frac{v_{m n}(\theta, \phi)}{444}\right]$

In above two expressions, $v_{m n}\left(\theta_{0}, \phi_{0}\right)$ and $v_{m n}(\theta, \phi)$ must be determined from $\beta_{m n}\left(\theta_{0}, \phi_{0}\right)$ and $\beta_{m n}(\theta, \phi)$ using (23), where $\psi(v)$ should be replaced by $\beta_{m n}\left(\theta_{0}, \phi_{0}\right)$ or $\beta_{m n}(\theta, \phi)$. A look-up table of (23) is needed for this task in computer simulation.
During switching, the phase shifter phase is

$$
\begin{aligned}
\beta_{m n}\left(\theta_{0}, \phi_{0}, \theta, \phi, t\right)= & \beta_{m n}\left(\theta_{0}, \phi_{0}\right)+\psi\left(v_{m n}\left(\theta_{0}, \phi_{0}, \theta, \phi, t\right)\right) \\
& 0 \leq t \leq t_{m n}-t_{0 m n} \\
= & \beta_{m n}\left(\theta_{0}, \phi_{0}\right)+\psi\left(t+t_{0 m n}\right) \\
& 0 \leq t \leq t_{m n}-t_{0 m n}
\end{aligned}
$$

where $\beta_{m n}\left(\theta_{0}, \phi_{0}\right)$ is given by (20).

During switching transient, observed from direction $(\theta, \phi)$, the $m n$th signal's phase consists of three parts: the phase due to feed and steering delay $\alpha_{\mathrm{mn}}^{\prime}(\theta, \phi)$, the phase shifter phase at initial position $\beta_{m n}\left(\theta_{0}, \phi_{0}\right)$, and the transient phase $\psi(t+$ $\left.t_{0 m n}\right)$. Thus, the total phase is

$$
\Phi_{m n}(t)=\alpha_{m n}^{\prime}(\theta, \phi)+\beta_{m n}\left(\theta_{0}, \phi_{0}\right)+\psi\left(t+t_{0 m n}\right) .
$$

The evaluation of this total phase involves (20), (7), (27), (30), and (26). After switching, the phase shifter's setting will reach the new value $\beta_{m n}(\theta, \phi)$ which compensates for $\operatorname{Mod}\left(\alpha_{m n}^{\prime}(\theta, \phi), 2 \pi\right)$ and consequently $\Phi_{m n}\left(t_{m n}\right)=0$.

Thus, the signal observed from direction $(\theta, \phi)$ is shown in (34) at the bottom of the page, where $\tau_{m n c}$ is calculated with $(\theta, \phi)$. For PSK and QAM, $\varphi\left(t-\tau_{m n c}\right)=\varphi$, is a constant in (34).

Note that from Fig. 6 it is seen that the phase transient time can be as long as several milliseconds. This could affect thousands even millions of data symbols, depending on the symbol rates. Fortunately, as described above, the number of large phase increments is only around $1 \%$ of the total. This makes the majority of phase increments, hence the majority of transient times, small. But still the transient time for those small ones can be in the order of $100 \mu \mathrm{s}$, which still covers a big number of symbols for high symbol rate transmission.

Simulation has been conducted to determine the extent of signal degradation, hence BER degradation, during switching.

\section{Methods of Evaluating PERformances}

To evaluate the BER of the reflectarray system, two methods are used and results are compared. The first method is a calculation method and the second method is the Monte Carlo simulation using randomly generated signal symbols. While the Monte Carlo simulation is well known and need not be described, the calculation method needs to be discussed. Parameters of Antenna 1 are used in all calculations and simulations.

With the calculation method, first, using any computer program, signal patterns of three consecutive symbols are generated. The total number of three-symbol patterns is $N=M^{3}$, for MPSK or M-ary QAM. Each and every one of all possible three-symbol patterns is used as the input signal to each of the 925 elements of Antenna 1. The output of Antenna 1 is formed as the sum of the signals reflected from 925 elements; each

$$
s(t)=\left\{\begin{array}{lc}
(\cos \theta) \sum_{m} \sum_{m} f(m, n) A\left(t-\tau_{m n c}\right) \cos \left[2 \pi f_{c}\left(t-\tau_{m n c}\right)+\varphi\left(t-\tau_{m n c}\right)+\Phi_{m n}(t)\right], & 0 \leq t \leq t_{m n}-t_{0 m n} \\
(\cos \theta) \sum_{m}^{m} \sum_{m} f(m, n) A\left(t-\tau_{m n c}\right) \cos \left[2 \pi f_{c}\left(t-\tau_{m n c}\right)+\varphi\left(t-\tau_{m n c}\right)\right], & t>t_{m n}-t_{0 m n}
\end{array}\right.
$$




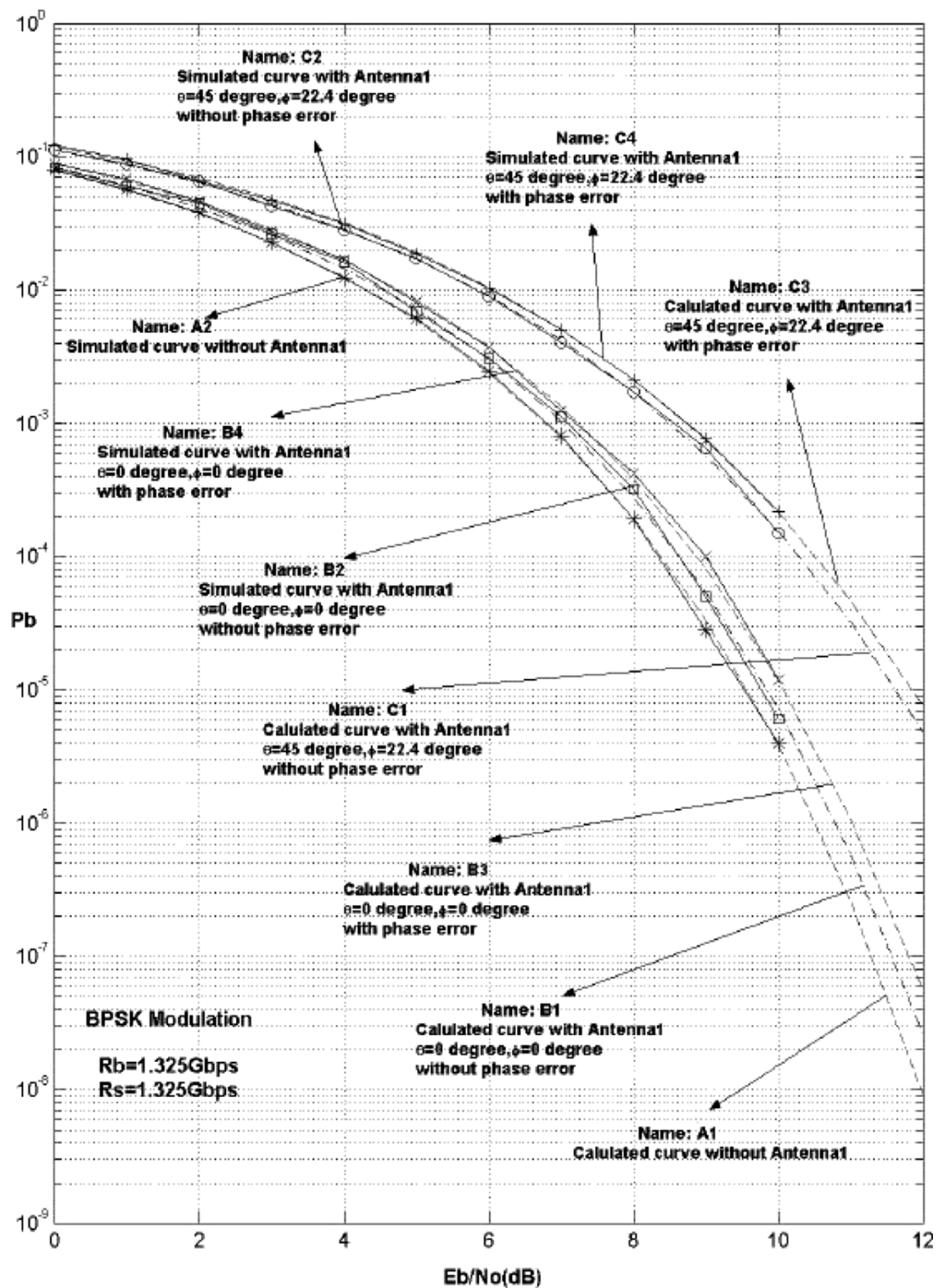

Fig. 7. BER curves of BPSK.

with a different delay and amplitude calculated according to the element's position coordinates $(m, n)$, using expression (18). Only the central symbol has been affected by two adjacent symbols. That is, only the central symbol is a complete reflectarray output symbol and can be used for BER evaluation. The central symbol of the output signal is sent to the coherent demodulator to correlate with the I- and Q-channel reference signals. The I- and Q-channel correlator outputs, $r_{I}$ and $r_{Q}$ are used to de- termine the signal energy as in $E=r_{I}^{2}+r_{Q}^{2}$ and phase as in $\theta=\arctan \left(r_{Q} / r_{I}\right)$.

Once $\mathrm{E}$ and $\theta$ are determined for symbol pattern $i$, they are used in the following integral to compute the symbol error probability for that particular symbol [7, p. 140]

$$
P_{s}(i)=1-\iint_{Z_{i}} p\left(x, y \mid s_{i}\right) d x d y
$$




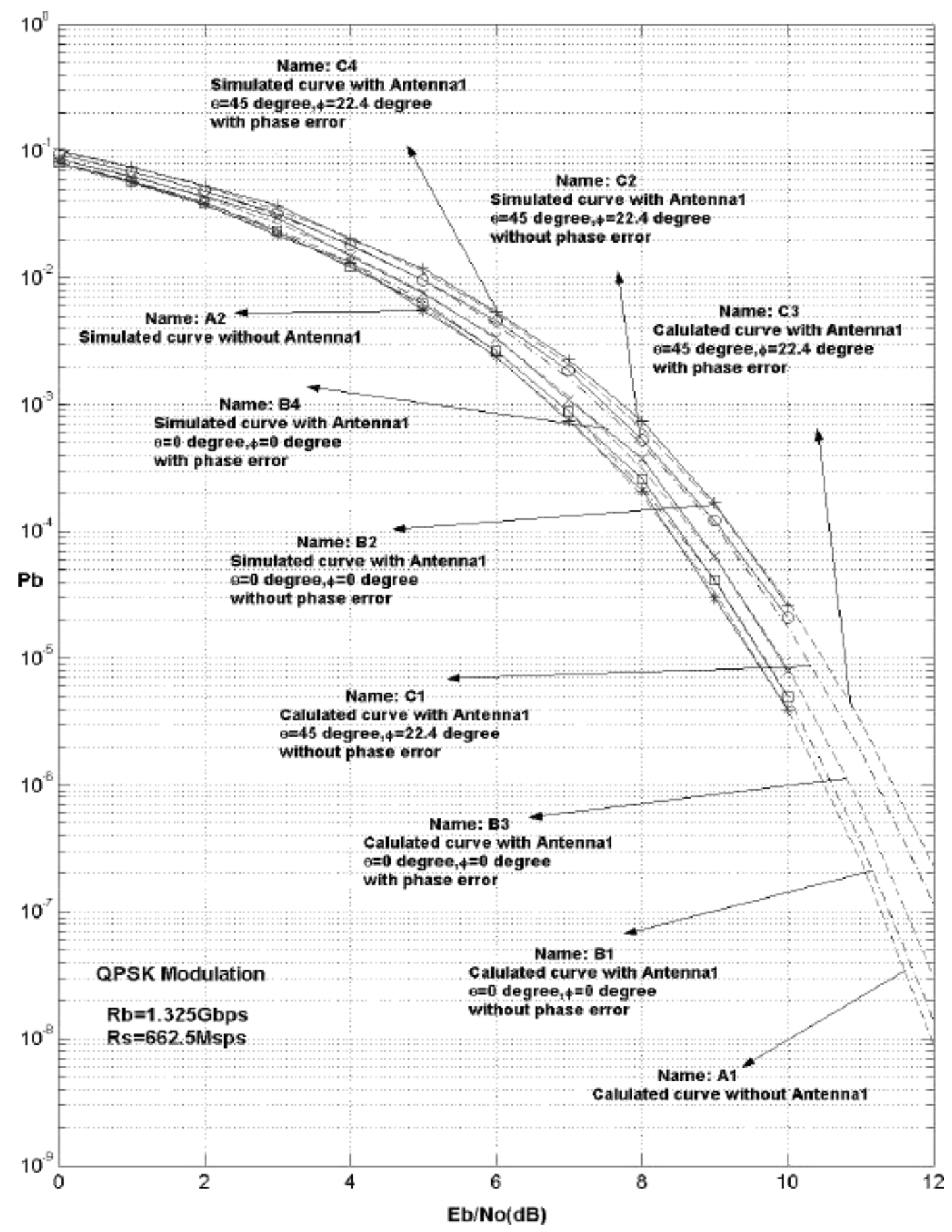

Fig. 8. BER curves of QPSK.

where $Z_{i}$ is the decision region for the symbol, and $p\left(x, y / s_{i}\right)$ is a conditional joint probability density function given by

$$
\begin{aligned}
& P\left(x, y \mid s_{i}\right)=\frac{1}{\pi N_{o}} \\
& \times \exp \left\{-\frac{1}{N_{o}}\left[\left(x-\sqrt{E_{i}} \cos \theta_{i}\right)^{2}+\left(x-\sqrt{E_{i}} \sin \theta_{i}\right)^{2}\right]\right\}
\end{aligned}
$$

where $E_{i}$ is the energy, and $\theta_{i}$ is the phase, of the central symbol $s_{i}$ of symbol pattern $i . N_{o}$ is the one-sided power spectral density of the additive white Gaussian noise in the system. The decision region $Z_{i}$ has the shape of a slice of a pie in an MPSK scheme, the angle of the slice is $2 \pi / M$. And in a QAM scheme, $Z_{i}$ is a square region determined by the constellation grid [7]. The final symbol error probability is the average of all $M^{3}$ three-symbol-patterns and BER is computed from the symbol error probability using appropriate conversion formulas [7].

In the following, results from the calculation method are presented as "calculated" curves and results from the Monte Carlo method are presented as "simulated" curves. In a few cases where the antenna is assumed ideal (no ISI and phase errors) "calculated" curves are obtained from existing theoretical expressions. 


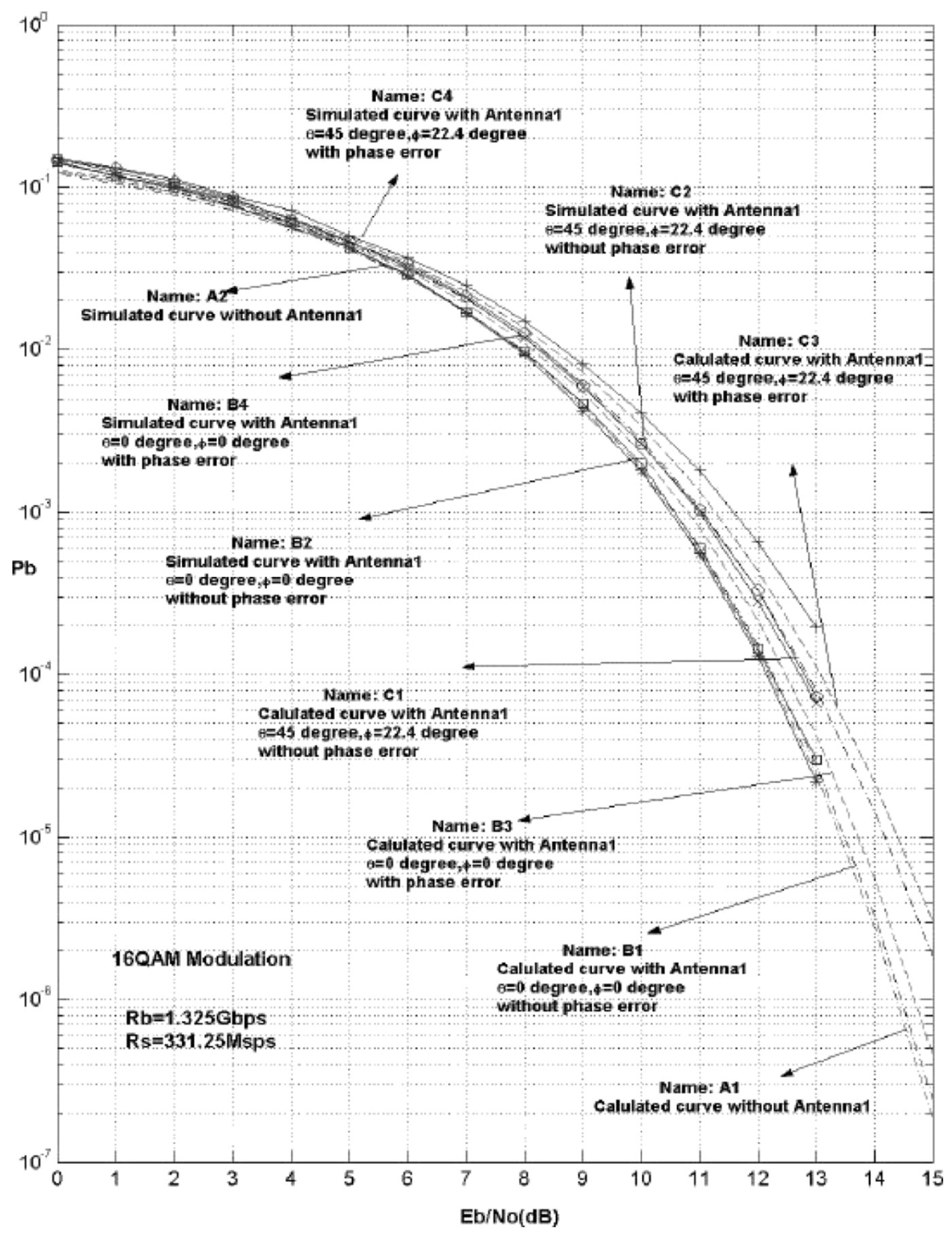

Fig. 9. BER curves of 16QAM.

\section{Simulation RESUlts On EFFECTS OF ISI AND RANDOM PHASE ERRORS}

The modulation schemes simulated are BPSK, QPSK, 8PSK, 16QAM, 64QAM, and 256QAM. The data bit rate is $1.325 \mathrm{G}$ bits/s and carrier frequency is $26.5 \mathrm{GHz}$. The bit rate was kept unchanged for all schemes, thus the symbol rate is $1.325 \mathrm{G} / \log _{2} M$ for an M-ary modulation.

For BPSK, QPSK, 8PSK, and 16QAM, BER curves are computed and simulated for the following representative cases as labeled in the Figs. 7-10, including
(A) "Without Antenna 1," which does not mean there is no antenna in the system, it merely assumes that the antenna is free from the three impairments investigated in this paper: ISI, phase shifters' static random errors, and transient phase errors. This is a hypothetical ideal case whose BER performance will serve as a benchmark. The BER curves in this case include A1: calculated curve without Antenna 1, and A2: simulated curve without Antenna 1. "Calculated curves" are used for comparison. The BER expressions for "calculated curves" of BPSK and QPSK can be found in [7] and 


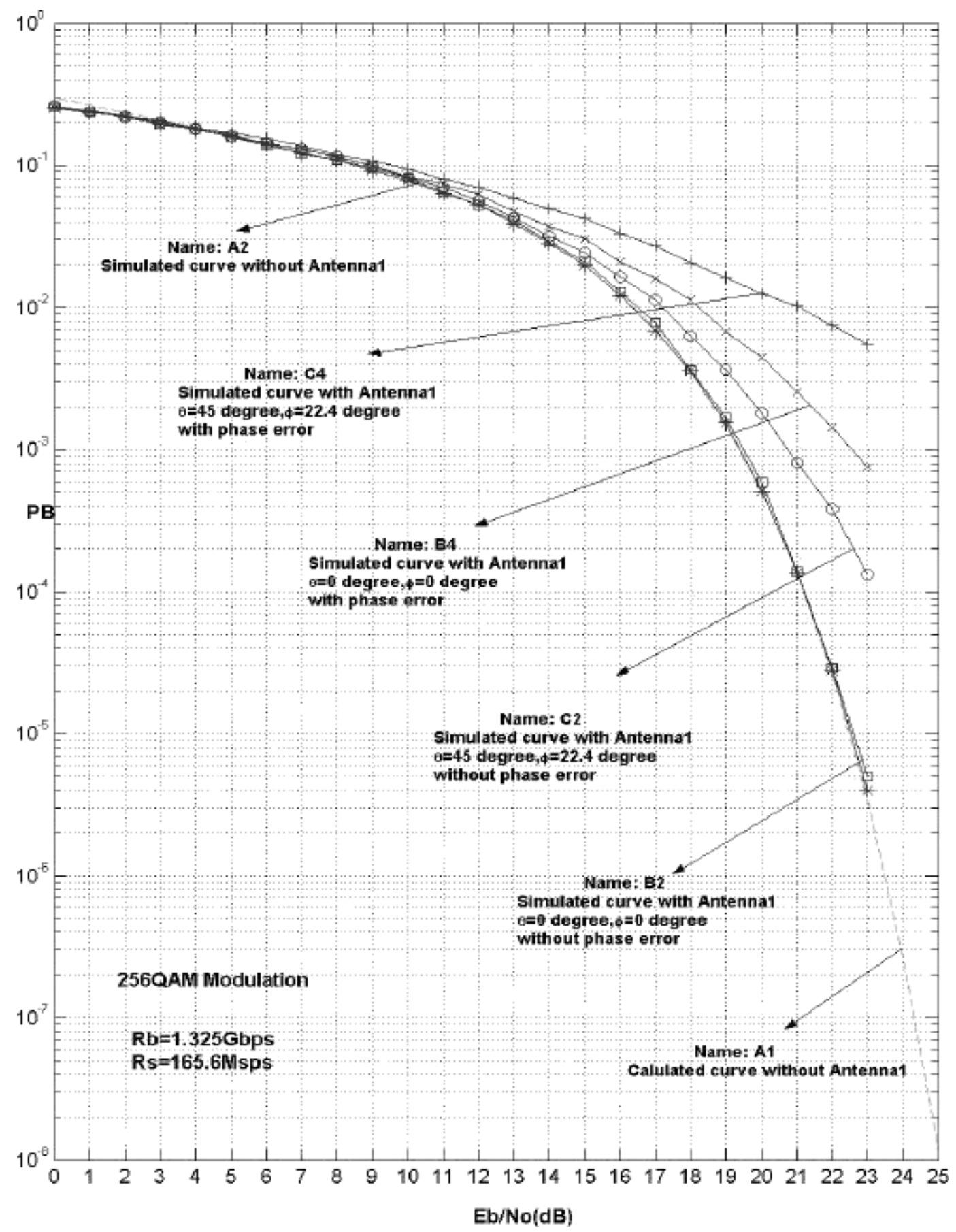

Fig. 10. BER curves of 256QAM.

those for higher order MPSK and QAM can be found in [8].

(B) "With Antenna 1, theta $(\theta)=0^{\circ}$, phi $(\phi)=0^{\circ}$," which is the case where Antenna 1 is pointing to the bore sight and the ISI loss is due to feed delay only. We call this case the best case. The BER curves in this case include four sub-cases which are "calculated curves:" B1, B3 and "simulated curves:" B2, B4 (see Fig. 7 for definitions of cases B1 to B4). In Figs. 7-10, the phrase "with phase error" means that the static random phase error of the phase shifters that is uniformly distributed in $[-\pi / 8, \pi / 8]$ is considered in the composite signal model.
(C) "With Antenna 1, theta $(\theta)=45^{\circ}$, phi $(\phi)=22.4^{\circ}$," which is the case where Antenna 1 is pointing to the direction where the ISI loss is the largest. We call this case the worst case. The BER curves in this case include sub-cases $\mathrm{C} 1$ to $\mathrm{C} 4$ (see Fig. 7 for definitions of cases $\mathrm{C} 1$ to $\mathrm{C} 4$ ).

For 64QAM and 256QAM, all above cases are simulated but only Case A1 is calculated due to the impractically long time of calculating the other curves. Simulations are performed to the extent that the BER is around $10^{-5}$. At this BER, the simulation time of the high-speed two-processor workstation for a single curve varies from several hours for the lowest order modulation (BPSK) to several days for the highest order modulation 


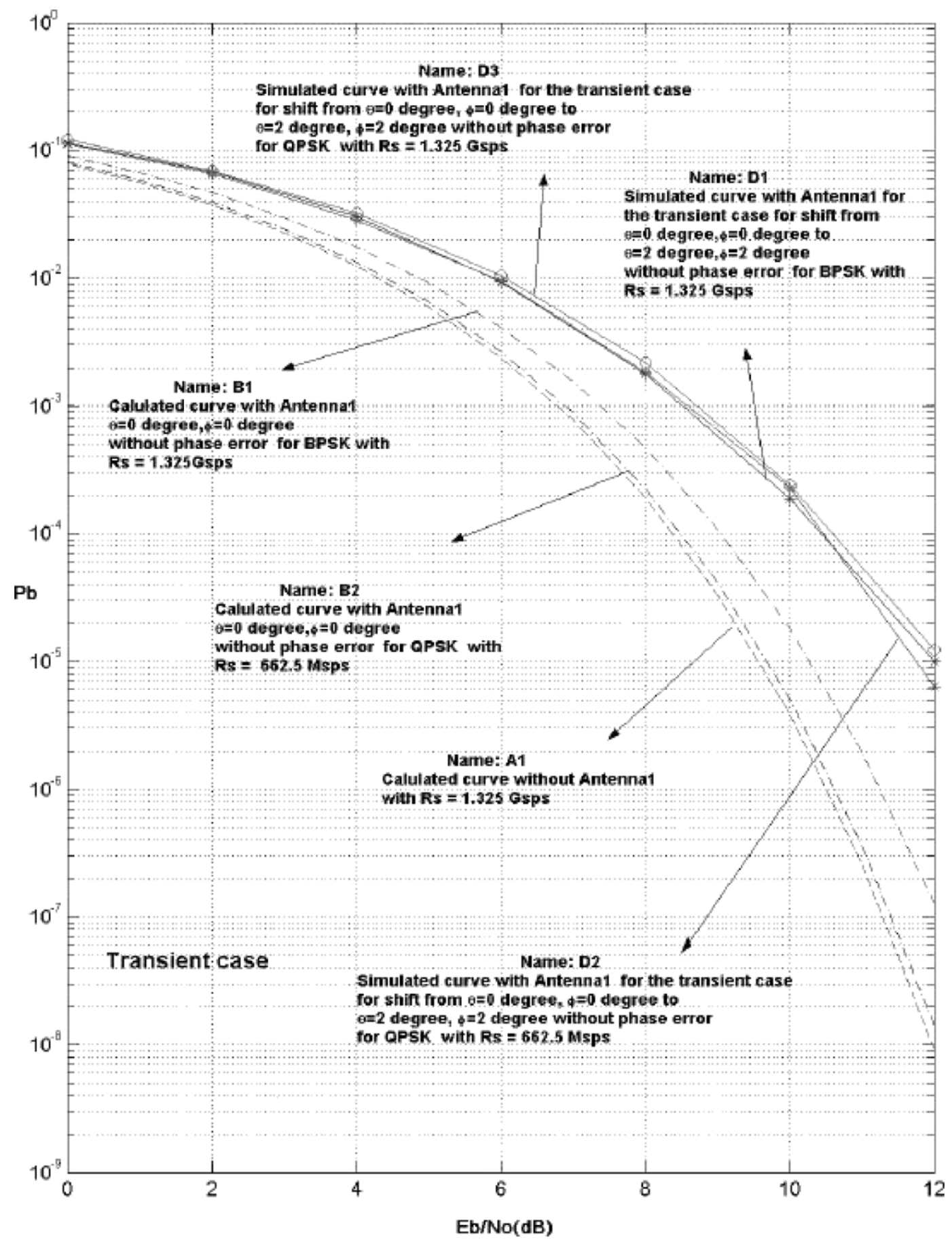

Fig. 11. BER curves of transient case for BPSK and QPSK.

(256QAM). For even lower BER, only the calculation results are given.

Due to the page limit, selected results for BPSK, QPSK, 16QAM, and 256QAM are presented in Figs. 7-10, where BPSK and QPSK are the most popular PSK schemes, 16QAM is the lowest order and a popular QAM scheme and 256QAM is the highest order QAM in practical use today (in computer modem only, though).

From all simulation results that we obtained, including those in Figs. 7-10 and the results for 8PSK and 64QAM that are not presented in figures, we have observed the following.
1. For all schemes simulated we see good agreement between the calculated curves and the simulated curves in cases where phase errors are absent (compare curves A1 to A2, B1 to B2, and C1 to C2 in Figs. 7-10).

2. We see various degradations for different cases. Comparing curves $\mathrm{B} 2$ to $\mathrm{A} 2$ (or B1 to A1), we see that in the best case without phase error, the BER degradation is very small, just a small fraction of a $\mathrm{dB}$. Comparing curve $\mathrm{C} 2$ to $\mathrm{A} 2$ (or $\mathrm{C} 1$ to $\mathrm{A} 1$ ) at $\mathrm{BER}=10^{-3}$, we see that in the worst case without phase error, the BER degradation is about $1.8,0.71,0.5,0.5,0.67$, and 1.25 
dB for BPSK, QPSK, 8PSK, 16QAM, 64QAM, and 256QAM, respectively. From these data we see that in general the ISI loss decreases as modulation order increases. This is due to the decrease in the ratio of ISI length over the symbol period. However, when the order is higher than around 16, the ISI loss starts to increase with the increase of the order since the ISI not only causes energy loss but also creates phase deviations in the composite signal, which causes loss in high-order modulations. This is more evident when phase shifters' phase error is considered, as demonstrated next.

3. In general, phase shifters' phase errors cause further degradation in BER. The simulation results show that it is about $0.2 \mathrm{~dB}$ for BPSK, QPSK, and 8PSK (figure not shown). This is very consistent with the theoretical analysis where only the average effect of phase errors, i.e., the amplitude reduction, is considered. For low-order modulations up to 8PSK, it is clear that only the amplitude reduction effect is evident while the net phase error is so small compared to the signal's own phases that its effect is not observed. However, the net phase error's effect is evident for 16QAM (an additional loss of about $0.5 \mathrm{~dB}$ at $\mathrm{BER}=10^{-3}$ ) and is very significant for very high-order modulations like 64QAM (an additional loss of about $1.6 \mathrm{~dB}$ at BER = $10^{-3}$ ) and 256QAM (an additional loss of about $5 \mathrm{~dB}$ at $\mathrm{BER}=5 \times 10^{-3}$ ). The reason that very high-order modulations like 64QAM and 256QAM behave so bad in the presence of phase errors is that the phase differences between symbols in 64QAM and 256 QAM are often very small, much smaller than the phase errors, so that the phase errors degrade the BER performance severely.

\section{Simulation Results on Effect of Phase Transient}

Simulation for transient state of the Antenna 1 has been performed using the formulas developed in Section V. To simulate the transient state, from Fig. 6 we can see that the longest time duration needed for the phase shifters to complete the phase changes of near $360^{\circ}$ is about $3 \mathrm{~ms}$. For a data rate of 1.325 Gbps, there are 3.975 million bits in these $3 \mathrm{~ms}$ to be simulated. Further, the complicated formulas in Section V for constructing the composite signal make the time to construct the composite signal much longer than that needed for the antenna's static state. These two factors make the simulation extremely time consuming.

We were able to simulate the BPSK and QPSK for the case of switching from $(0,0)$ direction to $(2,2)$ direction. By starting from $(0,0)$ direction, the ISI effect was kept at its minimum (only ISI due to feed delay exists). To isolate the effect of transient, the phase shifters random phase errors were not included. The net simulation time was about nine weeks for the three curves D1, D2, and D3, shown in Fig. 11.

From the figure we can see that compared to the ideal case (A1) the degradation due to switching transient for BPSK ranges from $1.8 \mathrm{~dB}$ at $\mathrm{BER}=10^{-3}$ to about $2.5 \mathrm{~dB}$ at $\mathrm{BER}=10^{-5}$.
The results for QPSK are similar. These results are quite consistent with the results from array factor calculation presented in Section V-C. Even though BER degradation is mainly due to energy loss caused by phase transient as demonstrated by array factor analysis and phase distortion's contribution to degradation is minor, for higher order modulations, we expect larger degradations due to phase distortion since higher order modulations are more susceptible to phase errors. This has been verified by the simulation of QPSK at the same symbol rate as that of BPSK (curve D3), where degradation is higher. When the symbol rate is reduced, the QPSK exhibits almost identical BER performance for the transient case (curve D2). This is because when symbol rate is lowered, the effect of phase distortion is reduced.

\section{CONCLUSION}

This paper presents analytical and simulation results for behaviors of several widely used digital modulations in systems equipped with reflectarray antennas. The formulas developed in this paper can be used for other similar reflectarray antennas.

The paper shows that ISI exists in the transmitted signal of the reflectarray and it degrades the BER performance. The phase shifter's phase errors incur further BER increase. The reflectarray's switching transient also causes BER increase. All three impairments' effects are severe with high-order modulations. Therefore low-order modulations like BPSK, QPSK, 8PSK, and 16QAM are preferred for reflectarray antennas. QPSK is the best among the above modulations since it doubles the data rate of BPSK within the same bandwidth while keeps the same BER performance in AWGN channel, and suffers less ISI loss with reflectarray. For Antenna 1, the total loss due to ISI and phase errors for QPSK is about $1 \mathrm{~dB}$ at BER $=10^{-3}$, which can be turned into $10^{-6}$ to $10^{-10}$ with typical error-control coding. This study shows that the example Antenna 1 reflectarray is operational for data rates up to $1 \mathrm{Gbps}$. However it is sensitive to phase shifters' phase errors for high-order modulations. For the reflectarray to be used for high order modulations, the phase errors must be kept very low. Phase transient during beam switching has relatively bigger impact on the BER degradation (about $2 \mathrm{~dB}$ for BPSK and QPSK). On one hand, this is not catastrophic, meaning the reflectarray can still maintain a decent BER performance even during beam switching; on the other hand, this loss is on top of the loss due to ISI and static phase errors, which makes the total loss for QPSK approaching $3 \mathrm{~dB}$ during switching. Therefore, the loss due to the phase transient should be made as small as possible by shortening the phase shifters' transient time.

\section{REFERENCES}

[1] D. Berry, R. Malech, and W. Kennedy, "The reflectarray antenna," IEEE Trans. Antennas Propag., vol. 11, no. 6, pp. 645-651, Nov. 1963.

[2] J. Huang and R. J. Pogorzelski, "A Ka-Band microstrip reflectarray with elements having variable rotation angles," IEEE Trans. Antennas Propag., vol. 46, no. 5, pp. 650-656, May 1998.

[3] R. Romanofsky et al., "Ka-Band phased array antennas based on BST thin film phase shifters," IEEE Trans. Microwave Theory Technol., vol. 48, no. 12, pp. 2504-2510, Dec. 2000.

[4] C. A. Balanis, Antenna Theory: Analysis and Design. New York: Harper \& Row, 1982. 
[5] J. Huang, "Analysis of a Microstrip Reflectarray Antenna for Microspacecraft Applications," JPL, CA, TDA Progress Rep. 42-120, Feb. 15, 1995.

[6] Y. T. Lo and S. W. Lee, Antenna Handbook. New York: Van Nostrand Reinhold, 1988.

[7] F. Xiong, Digital Modulation Techniques. Boston, MA: Artech House, 2000.

[8] J. Lu, K. Letaief, J. Chuang, and M. Liou, "M-PSK and M-QAM BER computation using signal-space concepts," IEEE Trans. Commun., vol. 47, no. 2, pp. 181-184, Feb. 1999.

[9] F. Xiong, Power efficient reflectarray communications system modulation study, in Final report to NASA Glenn Research Center, Jul. 17, 2003.

[10] R. Romanofsky and A. Qureshi, "A model for ferroelectric phase shifters," IEEE Trans. Magn., vol. 36, no. 5, pp. 3491-3494, Sep. 2000. 